\title{
Generalizations and applications of Young's integral inequality by higher order derivatives
}

Jun-Qing Wang ${ }^{1}$, Bai-Ni Guo ${ }^{2^{*}}$ (D) and Feng Qi ${ }^{3,4}$

\section{"Correspondence:}

bai.ni.guo@gmail.com

${ }^{2}$ School of Mathematics and Informatics, Henan Polytechnic University, Jiaozuo, China Full list of author information is available at the end of the article

\begin{abstract}
In the paper, the authors

1. generalize Young's integral inequality via Taylor's theorems in terms of higher order derivatives and their norms, and

2. apply newly-established integral inequalities to estimate several concrete definite integrals, including a definite integral of a function which plays an indispensable role in differential geometry and has a connection with the Lah numbers in combinatorics, the exponential integral, and the logarithmic integral.
\end{abstract}

MSC: Primary 26D15; secondary 26A42; 26A48; 26A51; 26D05; 26D07; 33B10; 33B20; $41 \mathrm{~A} 58$

Keywords: Young's integral inequality; Inverse function; Taylor's theorem; Higher order derivative; Lebesgue measure; Norm; Application; Exponential integral; Logarithmic integral; Existence of partitions of unity

\section{A concise retrospection}

In 1912, Young proved the following integral inequality in which an inverse function is involved.

Theorem 1.1 ([29]) Let $h(x)$ be a continuous and strictly increasing function on $[0, c]$ with $c>0$. If $h(0)=0, a \in[0, c]$, and $b \in[0, h(c)]$, then

$$
\int_{0}^{a} h(x) \mathrm{d} x+\int_{0}^{b} h^{-1}(x) \mathrm{d} x \geq a b,
$$

where $h^{-1}$ is the inverse function of $h$. The equality in (1.1) is valid if and only if $b=h(a)$.

For avoiding confusion, we call inequality (1.1) Young's integral inequality, because several other inequalities, such as

$$
\sum_{k=1}^{n} \frac{\cos (k \theta)}{k}>-1, \quad n \geq 2, \theta \in[0, \pi]
$$

and the weighted arithmetic-geometric mean inequality, are also called Young's inequality.

(c) The Author(s) 2019. This article is distributed under the terms of the Creative Commons Attribution 4.0 International License (http://creativecommons.org/licenses/by/4.0/), which permits unrestricted use, distribution, and reproduction in any medium, provided you give appropriate credit to the original author(s) and the source, provide a link to the Creative Commons license, and indicate if changes were made. 
In [9, Secton 2.7] and [10, Chapter XIV], many extensions, refinements, generalizations, and applications of Young's integral inequality (1.1) in Theorem 1.1 were collected and reviewed.

In 2008, Hoorfar and Qi obtained the following double inequality which refines Young's integral inequality (1.1).

Theorem 1.2 ([4]) Let $h(x)$ be a differentiable and strictly increasing function on $[0, c]$ for $c>0$, and let $h^{-1}$ be the inverse function of $h$. If $h(0)=0, a \in[0, c], b \in[0, h(c)]$, and $h^{\prime}(x)$ is strictly monotonic on $[0, c]$, then

$$
\frac{m}{2}\left[a-h^{-1}(b)\right]^{2} \leq \int_{0}^{a} h(x) \mathrm{d} x+\int_{0}^{b} h^{-1}(x) \mathrm{d} x-a b \leq \frac{M}{2}\left[a-h^{-1}(b)\right]^{2},
$$

where

$$
m=\min \left\{h^{\prime}(a), h^{\prime}\left(h^{-1}(b)\right)\right\} \quad \text { and } \quad M=\max \left\{h^{\prime}(a), h^{\prime}\left(h^{-1}(b)\right)\right\} .
$$

The equalities in (1.2) are valid if and only if $b=h(a)$.

In 2009 and 2010, motivated by Theorem 1.2 and its proof in [4], among other things, Jakšetić and Pečarić extended and generalized Young's integral inequality (1.1) and Hoorfar-Qi's double inequality (1.2) as the following theorems.

Theorem 1.3 ([5, Theorem 2.1] and [6, Theorem 2.3]) Let $h(x)$ be a differentiable and strictly increasing function on $[0, c]$ for $c>0, h(0)=0, a \in[0, c], b \in[0, h(c)]$, and $h^{-1}$ be the inverse function of $h$. Denote

$$
\alpha=\min \left\{a, h^{-1}(b)\right\} \text { and } \beta=\max \left\{a, h^{-1}(b)\right\} .
$$

1. If $h^{\prime}(x)$ is increasing on $[\alpha, \beta]$ and $b<h(a)$, or if $h^{\prime}(x)$ is decreasing on $[\alpha, \beta]$ and $b>h(a)$, then

$$
\begin{aligned}
{\left[a-h^{-1}(b)\right]\left[h\left(\frac{a+h^{-1}(b)}{2}\right)-b\right] } & \leq \int_{0}^{a} h(x) \mathrm{d} x+\int_{0}^{b} h^{-1}(x) \mathrm{d} x-a b \\
& \leq \frac{1}{2}\left[a-h^{-1}(b)\right][h(a)-b] .
\end{aligned}
$$

2. If $h^{\prime}(x)$ is increasing on $[\alpha, \beta]$ and $b>h(a)$, or if $h^{\prime}(x)$ is decreasing on $[\alpha, \beta]$ and $b<h(a)$, then inequality (1.4) is reversed.

3. The equality in (1.4) is valid if and only if $h(x)=\lambda x$ for $\lambda>0$ or $b=h(a)$.

Theorem 1.4 ([6, Theorem 2.6]) Let $h(x)$ be a differentiable and strictly increasing function on $[0, c]$ for $c>0$, and let $h^{-1}$ be the inverse function of $h$. If $h(0)=0, a \in[0, c]$, 
$b \in[0, h(c)]$, and $h^{\prime}(x)$ is convex on $[\alpha, \beta]$, then

$$
\begin{aligned}
\frac{\left[a-h^{-1}(b)\right]^{2}}{2} h^{\prime}\left(\frac{a+2 h^{-1}(b)}{3}\right) & \leq \int_{0}^{a} h(x) \mathrm{d} x+\int_{0}^{b} h^{-1}(x) \mathrm{d} x-a b \\
& \leq \frac{\left[a-h^{-1}(b)\right]^{2}}{3}\left[\frac{h^{\prime}(a)}{2}+h^{\prime}\left(h^{-1}(b)\right)\right] .
\end{aligned}
$$

If $h^{\prime}(x)$ is concave, then the double inequality (1.5) is reversed.

Theorem 1.5 ([6, Theorem 2.1]) Let $h(x)$ be a differentiable and strictly increasing function on $[0, c]$ for $c>0$, and let $h^{-1}$ be the inverse function of $h$. If $h(0)=0, a \in[0, c]$, $b \in[0, h(c)]$, and $h^{\prime}(x)$ is almost everywhere continuous with respect to Lebesgue measure on $[\alpha, \beta]$, then the double inequality

$$
C_{u}\left\|h^{\prime}\right\|_{v} \leq \int_{0}^{a} h(x) \mathrm{d} x+\int_{0}^{b} h^{-1}(x) \mathrm{d} x-a b \leq C_{p}\left\|h^{\prime}\right\|_{q}
$$

is valid for all $u, v$ and $p, q$ satisfying

1. $\frac{1}{u}+\frac{1}{v}=1$ for $u, v \in(-\infty, 0) \cup(0,1)$, or $(u, v)=(1,-\infty)$, or $(u, v)=(-\infty, 1)$;

2. $\frac{1}{p}+\frac{1}{q}=1$ for $1<p, q<\infty$, or $(p, q)=(+\infty, 1)$, or $(p, q)=(1,+\infty)$;

where

$$
C_{r}= \begin{cases}{\left[\frac{\mid a-h^{-1}(b) r^{r+1}}{r+1}\right]^{1 / r},} & r \neq 0, \pm \infty ; \\ \left|a-h^{-1}(b)\right|, & r=+\infty ; \\ 0, & r=-\infty\end{cases}
$$

and

$$
\left\|h^{\prime}\right\|_{r}= \begin{cases}{\left[\int_{\alpha}^{\beta}\left[h^{\prime}(t)\right]^{r} \mathrm{~d} t\right]^{1 / r},} & r \neq 0, \pm \infty \\ \sup \left\{h^{\prime}(t), t \in[\alpha, \beta]\right\}, & r=+\infty ; \\ \inf \left\{h^{\prime}(t), t \in[\alpha, \beta]\right\}, & r=-\infty .\end{cases}
$$

We note that Hoorfar-Qi's double inequality (1.2) has been applied and employed in the paper [7] and in the Undergraduate Texts in Mathematics [8].

In this paper, by virtue of Taylor's theorems with different remainders, we establish some integral inequalities of Hoorfar-Qi's type in terms of higher order derivatives and their norms, demonstrate that these newly-established integral inequalities generalize Young's integral inequality (1.1), Hoorfar-Qi's integral inequality (1.2), and Jakšetić-Pečarić's integral inequalities (1.4), (1.5), and (1.6), and apply these integral inequalities to estimate several concrete definite integrals, including a definite integral of $e^{-1 / x}$ which plays an indispensable role in differential geometry and has a connection with the Lah numbers in combinatorics, the exponential integral $\operatorname{Ei}(x)$, and the logarithmic integral $\operatorname{li}(x)$.

\section{Lemmas}

For proving our main result, we need Taylor's theorems below. 
Lemma 2.1 ([2, p. 113, Theorem 5.19]) Let $f(x)$ be a function having finite nth derivative $f^{(n)}(x)$ everywhere in an open interval $(\mu, v)$ and assume that $f^{(n-1)}(x)$ is continuous on the closed interval $[\mu, \nu]$. Then, for a fixed point $x_{0} \in[\mu, v]$ and every $x \in[\mu, \nu]$ with $x \neq x_{0}$, there exists a point $x_{1}$ interior to the interval joining $x$ and $x_{0}$ such that

$$
f(x)=f\left(x_{0}\right)+\sum_{k=1}^{n-1} \frac{f^{(k)}\left(x_{0}\right)}{k !}\left(x-x_{0}\right)^{k}+\frac{f^{(n)}\left(x_{1}\right)}{n !}\left(x-x_{0}\right)^{n} .
$$

Lemma 2.2 ([1, p. 279, Theorem 7.6] and [11, p. 6, 1.4.37]) If $f(x) \in C^{n+1}[\mu, \nu]$ and $x_{0} \in$ $[\mu, v]$, then

$$
f(x)=\sum_{k=0}^{n} \frac{f^{(k)}\left(x_{0}\right)}{k !}\left(x-x_{0}\right)^{k}+\frac{1}{n !} \int_{x_{0}}^{x}(x-t)^{n} f^{(n+1)}(t) \mathrm{d} t .
$$

For proving our main results, we also need Hölder's integral inequality and its reversed version, Čebyšev's integral inequality, discrete and integral versions of Jensen's inequality, and Hermite-Hadamard's integral inequality.

Lemma 2.3 ([10, Chapter V] and [23-25]) Let $\frac{1}{p}+\frac{1}{q}=1$ with $p>0$ and $p \neq 1$, let $f$ and $g$ be real functions on $[\mu, \nu]$, and let $|f|^{p}$ and $|g|^{q}$ be integrable on $[\mu, \nu]$.

1. If $p>1$, then

$$
\int_{\mu}^{v}|f(x) g(x)| \mathrm{d} x \leq\left[\int_{\mu}^{v}|f(x)|^{p} \mathrm{~d} x\right]^{1 / p}\left[\int_{\mu}^{v}|g(x)|^{q} \mathrm{~d} x\right]^{1 / q} .
$$

The equality in (2.3) holds if and only if $A|f(x)|^{p}=B|g(x)|^{q}$ almost everywhere for two constants $A$ and $B$.

2. If $p<1$ and $p \neq 0$, then inequality (2.3) is reversed.

Lemma 2.4 ([10, Chapter IX] and [19]) Let $f, g:[\mu, \nu] \rightarrow \mathbb{R}$ be integrable functions satisfying that they are both increasing or both decreasing. Then

$$
\int_{\mu}^{v} f(x) \mathrm{d} x \int_{\mu}^{\nu} g(x) \mathrm{d} x \leq(\nu-\mu) \int_{\mu}^{\nu} f(x) g(x) \mathrm{d} x .
$$

If one of the functions $f$ or $g$ is nonincreasing and the other nondecreasing, then the inequality in (2.4) is reversed.

Lemma 2.5 ([9, Sect. 1.4] and [10, Chapter I]) Iff is a convex function on an interval $I \subseteq \mathbb{R}$ and if $n \geq 2$ and $x_{k} \in I$ for $1 \leq k \leq n$, then

$$
f\left(\frac{1}{\sum_{k=1}^{n} p_{k}} \sum_{k=1}^{n} p_{k} x_{k}\right) \leq \frac{1}{\sum_{k=1}^{n} p_{k}} \sum_{k=1}^{n} p_{k} f\left(x_{k}\right),
$$

where $p_{k}>0$ for $1 \leq k \leq n$. Iff is concave, inequality (2.5) is reversed. 
Let $\phi$ be a convex function on $[\mu, \nu], f \in L_{1}(\mu, v)$, and $\sigma$ be a nonnegative measure. Then

$$
\phi\left(\frac{\int_{\mu}^{v} f(x) \mathrm{d} \sigma}{\int_{\mu}^{v} \mathrm{~d} \sigma}\right) \leq \frac{\int_{\mu}^{v} \phi(f(x)) \mathrm{d} \sigma}{\int_{\mu}^{v} \mathrm{~d} \sigma} .
$$

If $\phi$ is a concave function, then inequality (2.6) is reversed.

These five lemmas are general knowledge in mathematics and they will continue to play important roles in this paper.

Lemma 2.6 ([12, 26-28]) Let $f(x)$ and $g(x)$ be nonnegative and convex functions on $[\mu, \nu]$. Then

$$
\begin{aligned}
& 2 f\left(\frac{\mu+v}{2}\right) g\left(\frac{\mu+v}{2}\right)-\frac{1}{6} M(\mu, v)-\frac{1}{3} N(\mu, v) \\
& \quad \leq \frac{1}{v-\mu} \int_{\mu}^{v} f(x) g(x) \mathrm{d} x \leq \frac{1}{3} M(\mu, v)+\frac{1}{6} N(\mu, v),
\end{aligned}
$$

where

$$
M(\mu, \nu)=f(\mu) g(\mu)+f(v) g(v) \quad \text { and } \quad N(\mu, v)=f(\mu) g(\nu)+f(v) g(\mu) .
$$

\section{Main results and proofs}

Now we are in a position to state and prove our main results.

Theorem 3.1 Let $h(0)=0$ and $h(x)$ be strictly increasing on $[0, c]$ for $c>0$, let $h^{(n)}(x)$ for $n \geq 0$ be continuous on $[0, c]$, let $h^{(n+1)}(x)$ be finite and strictly monotonic on $(0, c)$, and let $h^{-1}$ be the inverse function of $h$. For $a \in[0, c]$ and $b \in[0, h(c)]$,

1. if $b<h(a)$, then

$$
\begin{aligned}
& \sum_{k=1}^{n} h^{(k)}\left(h^{-1}(b)\right) \frac{\left[a-h^{-1}(b)\right]^{k+1}}{(k+1) !}+m_{n}(a, b) \frac{\left[a-h^{-1}(b)\right]^{n+2}}{(n+2) !} \\
& \quad \leq \int_{0}^{a} h(x) \mathrm{d} x+\int_{0}^{b} h^{-1}(x) \mathrm{d} x-a b \\
& \quad \leq \sum_{k=1}^{n} h^{(k)}\left(h^{-1}(b)\right) \frac{\left[a-h^{-1}(b)\right]^{k+1}}{(k+1) !}+M_{n}(a, b) \frac{\left[a-h^{-1}(b)\right]^{n+2}}{(n+2) !},
\end{aligned}
$$

where

$$
m_{n}(a, b)=\min \left\{h^{(n+1)}\left(h^{-1}(b)\right), h^{(n+1)}(a)\right\}
$$

and

$$
M_{n}(a, b)=\max \left\{h^{(n+1)}\left(h^{-1}(b)\right), h^{(n+1)}(a)\right\}
$$

2. if $b>h(a)$, then

(a) when $n=2 \ell$ for $\ell \geq 0$, the double inequality (3.1) is valid; 
(b) when $n=2 \ell+1$ for $\ell \geq 0$, we have

$$
\begin{aligned}
& \sum_{k=1}^{n} h^{(k)}\left(h^{-1}(b)\right) \frac{\left[a-h^{-1}(b)\right]^{k+1}}{(k+1) !}-M_{n}(a, b) \frac{\left[a-h^{-1}(b)\right]^{n+2}}{(n+2) !} \\
& \quad \leq \int_{0}^{a} h(x) \mathrm{d} x+\int_{0}^{b} h^{-1}(x) \mathrm{d} x-a b \\
& \quad \leq \sum_{k=1}^{n} h^{(k)}\left(h^{-1}(b)\right) \frac{\left[a-h^{-1}(b)\right]^{k+1}}{(k+1) !}-m_{n}(a, b) \frac{\left[a-h^{-1}(b)\right]^{n+2}}{(n+2) !}
\end{aligned}
$$

3. if, and only if, $b=h(a)$, those equalities in (3.1) and (3.2) hold.

Proof Employing several basic properties of definite integrals, such as substitution of variables and integration by parts, reveals

$$
\begin{aligned}
& \int_{0}^{a} h(x) \mathrm{d} x+\int_{0}^{b} h^{-1}(x) \mathrm{d} x \\
& \quad=\int_{0}^{a} h(x) \mathrm{d} x+\int_{0}^{h^{-1}(b)} y \mathrm{~d} h(y) \\
& \quad=\int_{0}^{a} h(x) \mathrm{d} x+\left.y h(y)\right|_{0} ^{h^{-1}(b)}-\int_{0}^{h^{-1}(b)} h(y) \mathrm{d} y=b h^{-1}(b)+\int_{h^{-1}(b)}^{a} h(y) \mathrm{d} y \\
& =a b+\int_{h^{-1}(b)}^{a}[h(y)-b] \mathrm{d} y=a b+\int_{h^{-1}(b)}^{a}\left[h(y)-h\left(h^{-1}(b)\right)\right] \mathrm{d} y .
\end{aligned}
$$

By virtue of formula (2.1), we have

$$
\begin{aligned}
h(y)-h\left(h^{-1}(b)\right)= & \sum_{k=1}^{n} \frac{h^{(k)}\left(h^{-1}(b)\right)}{k !}\left[y-h^{-1}(b)\right]^{k} \\
& +\frac{h^{(n+1)}(\xi)}{(n+1) !}\left[y-h^{-1}(b)\right]^{n+1},
\end{aligned}
$$

where $\xi$ is a point interior to the interval joining $y$ and $h^{-1}(b)$. As a result,

$$
\begin{aligned}
& \int_{h^{-1}(b)}^{a}\left[h(y)-h\left(h^{-1}(b)\right)\right] \mathrm{d} y \\
& =\sum_{k=1}^{n} \frac{h^{(k)}\left(h^{-1}(b)\right)}{k !} \int_{h^{-1}(b)}^{a}\left[y-h^{-1}(b)\right]^{k} \mathrm{~d} y \\
& \quad+\frac{1}{(n+1) !} \int_{h^{-1}(b)}^{a} h^{(n+1)}(\xi)\left[y-h^{-1}(b)\right]^{n+1} \mathrm{~d} y \\
& =\sum_{k=1}^{n} h^{(k)}\left(h^{-1}(b)\right) \frac{\left[a-h^{-1}(b)\right]^{k+1}}{(k+1) !}+\int_{h^{-1}(b)}^{a} h^{(n+1)}(\xi) \frac{\left[y-h^{-1}(b)\right]^{n+1}}{(n+1) !} \mathrm{d} y .
\end{aligned}
$$

When $h^{-1}(b)<a$, if $h^{(n+1)}(x)$ is strictly increasing on $(0, c)$, then

$$
h^{(n+1)}\left(h^{-1}(b)\right)<h^{(n+1)}(\xi)<h^{(n+1)}(y) \leq h^{(n+1)}(a)
$$


if $h^{(n+1)}(x)$ is strictly decreasing on $(0, c)$, then

$$
h^{(n+1)}(a) \leq h^{(n+1)}(y)<h^{(n+1)}(\xi)<h^{(n+1)}\left(h^{-1}(b)\right) .
$$

Consequently, the double inequality $m_{n}(a, b)<h^{(n+1)}(\xi)<M_{n}(a, b)$ is valid. Thus, it follows that

$$
\begin{aligned}
m_{n}(a, b) \frac{\left[a-h^{-1}(b)\right]^{n+2}}{(n+2) !} & \leq \int_{h^{-1}(b)}^{a} h^{(n+1)}(\xi) \frac{\left[y-h^{-1}(b)\right]^{n+1}}{(n+1) !} \mathrm{d} y \\
& \leq M_{n}(a, b) \frac{\left[a-h^{-1}(b)\right]^{n+2}}{(n+2) !} .
\end{aligned}
$$

Hence, the double inequality (3.1) is proved.

When $h^{-1}(b)>a$, if $h^{(n+1)}(x)$ is increasing on $(0, c)$, then

$$
h^{(n+1)}\left(h^{-1}(b)\right) \geq h^{(n+1)}(\xi) \geq h^{(n+1)}(y) \geq h^{(n+1)}(a)
$$

if $h^{(n+1)}(x)$ is decreasing on $(0, c)$, then

$$
h^{(n+1)}(a) \geq h^{(n+1)}(y) \geq h^{(n+1)}(\xi) \geq h^{(n+1)}\left(h^{-1}(b)\right) .
$$

Consequently, the double inequality $m_{n}(a, b)<h^{(n+1)}(\xi)<M_{n}(a, b)$ is still valid. Hence, since

$$
\begin{aligned}
& \int_{h^{-1}(b)}^{a} h^{(n+1)}(\xi) \frac{\left[y-h^{-1}(b)\right]^{n+1}}{(n+1) !} \mathrm{d} y \\
& \quad=\frac{(-1)^{n}}{(n+1) !} \int_{a}^{h^{-1}(b)} h^{(n+1)}(\xi)\left[h^{-1}(b)-y\right]^{n+1} \mathrm{~d} y,
\end{aligned}
$$

we acquire

$$
\begin{aligned}
\frac{(-1)^{n} m_{n}(a, b)}{(n+2) !}\left[h^{-1}(b)-a\right]^{n+2} & \leq \int_{h^{-1}(b)}^{a} h^{(n+1)}(\xi) \frac{\left[y-h^{-1}(b)\right]^{n+1}}{(n+1) !} \mathrm{d} y \\
& \leq \frac{(-1)^{n} M_{n}(a, b)}{(n+2) !}\left[h^{-1}(b)-a\right]^{n+2}, \quad n=2 \ell,
\end{aligned}
$$

and

$$
\begin{aligned}
\frac{(-1)^{n} m_{n}(a, b)}{(n+2) !}\left[h^{-1}(b)-a\right]^{n+2} & \geq \int_{h^{-1}(b)}^{a} h^{(n+1)}(\xi) \frac{\left[y-h^{-1}(b)\right]^{n+1}}{(n+1) !} \mathrm{d} y \\
& \geq \frac{(-1)^{n} M_{n}(a, b)}{(n+2) !}\left[h^{-1}(b)-a\right]^{n+2}, \quad n=2 \ell+1
\end{aligned}
$$

for $\ell \geq 0$. The double inequality (3.2) is thus proved. The proof of Theorem 3.1 is complete.

Remark 3.1 Taking $n=0$ in Theorem 3.1 leads to the above Theorem 1.2 in the paper [4]. 
Theorem 3.2 Let $n \geq 0$ and $h(x) \in C^{n+1}[0, c]$ such that $h(0)=0, h^{(n+1)}(x) \geq 0$ on $[\alpha, \beta]$, and $h(x)$ is strictly increasing on $[0, c]$ for $c>0$, let $h^{-1}$ be the inverse function of $h$, and let $a \in[0, c]$ and $b \in[0, h(c)]$. Then

1. when $b>h(a)$ and $n=2 \ell$ for $\ell \geq 0$ or when $b<h(a)$, we have

$$
\begin{aligned}
\frac{C_{u, n}}{(n+1) !}\left\|h^{(n+1)}\right\|_{\nu} \leq & \int_{0}^{a} h(x) \mathrm{d} x+\int_{0}^{b} h^{-1}(x) \mathrm{d} x-a b \\
& -\sum_{k=1}^{n} h^{(k)}\left(h^{-1}(b)\right) \frac{\left[a-h^{-1}(b)\right]^{k+1}}{(k+1) !} \leq \frac{C_{p, n}}{(n+1) !}\left\|h^{(n+1)}\right\|_{q} ;
\end{aligned}
$$

2. when $b>h(a)$ and $n=2 \ell+1$ for $\ell \geq 0$, we have

$$
\begin{aligned}
-\frac{C_{p, n}}{(n+1) !}\left\|h^{(n+1)}\right\|_{q} \leq & \int_{0}^{a} h(x) \mathrm{d} x+\int_{0}^{b} h^{-1}(x) \mathrm{d} x-a b \\
& -\sum_{k=1}^{n} h^{(k)}\left(h^{-1}(b)\right) \frac{\left[a-h^{-1}(b)\right]^{k+1}}{(k+1) !} \leq-\frac{C_{u, n}}{(n+1) !}\left\|h^{(n+1)}\right\|_{v} ;
\end{aligned}
$$

where $\alpha, \beta$ are defined as in (1.3),

$$
\begin{gathered}
C_{r, n}= \begin{cases}{\left[\frac{\mid a-h^{-1}(b) r^{r(n+1)+1}}{r(n+1)+1}\right]^{1 / r},} & r \neq 0, \pm \infty ; \\
\left|a-h^{-1}(b)\right|^{n+1}, & r=+\infty ; \\
0, & r=-\infty,\end{cases} \\
\left\|h^{(n+1)}\right\|_{r}= \begin{cases}{\left[\int_{\alpha}^{\beta}\left[h^{(n+1)}(t)\right]^{r} \mathrm{~d} t\right]^{1 / r},} & r \neq 0, \pm \infty ; \\
\sup \left\{h^{(n+1)}(t), t \in[\alpha, \beta]\right\}, & r=+\infty ; \\
\inf \left\{h^{(n+1)}(t), t \in[\alpha, \beta]\right\}, & r=-\infty,\end{cases}
\end{gathered}
$$

and $u, v, p, q$ satisfy

1. $u<1$ and $u \neq 0$ with $\frac{1}{u}+\frac{1}{v}=1$, or $(u, v)=(-\infty, 1)$, or $(u, v)=(1,-\infty)$;

2. $1<p, q<\infty$ with $\frac{1}{p}+\frac{1}{q}=1$, or $(p, q)=(+\infty, 1)$, or $(p, q)=(1,+\infty)$.

Proof Applying formula (2.2) to the last term in (3.3) yields

$$
\begin{aligned}
& \int_{0}^{a} h(x) \mathrm{d} x+\int_{0}^{b} h^{-1}(x) \mathrm{d} x-a b \\
& \quad=\sum_{k=1}^{n} h^{(k)}\left(h^{-1}(b)\right) \frac{\left[a-h^{-1}(b)\right]^{k+1}}{(k+1) !}+\int_{h^{-1}(b)}^{a} \frac{1}{n !} \int_{h^{-1}(b)}^{x}(x-t)^{n} h^{(n+1)}(t) \mathrm{d} t \mathrm{~d} x \\
& =\sum_{k=1}^{n} h^{(k)}\left(h^{-1}(b)\right) \frac{\left[a-h^{-1}(b)\right]^{k+1}}{(k+1) !}+\int_{h^{-1}(b)}^{a} \frac{1}{n !} \int_{t}^{a}(x-t)^{n} h^{(n+1)}(t) \mathrm{d} x \mathrm{~d} t \\
& =\sum_{k=1}^{n} h^{(k)}\left(h^{-1}(b)\right) \frac{\left[a-h^{-1}(b)\right]^{k+1}}{(k+1) !}+\int_{h^{-1}(b)}^{a} \frac{(a-t)^{n+1} h^{(n+1)}(t)}{(n+1) !} \mathrm{d} t .
\end{aligned}
$$


It is not difficult to verify that

$$
\int_{h^{-1}(b)}^{a}(a-t)^{n+1} h^{(n+1)}(t) \mathrm{d} t= \begin{cases}\int_{\alpha}^{\beta}|a-t|^{n+1} h^{(n+1)}(t) \mathrm{d} t, & b<h(a) ; \\ (-1)^{n} \int_{\alpha}^{\beta}|a-t|^{n+1} h^{(n+1)}(t) \mathrm{d} t, & b>h(a) .\end{cases}
$$

Since

$$
\begin{aligned}
\int_{\alpha}^{\beta}|a-t|^{n+1} h^{(n+1)}(t) \mathrm{d} t & \leq\left|a-f^{-1}(b)\right|^{n+1} \int_{\alpha}^{\beta} h^{(n+1)}(t) \mathrm{d} t \\
& =\left|a-f^{-1}(b)\right|^{n+1}\left\|h^{(n+1)}\right\|_{1}, \\
\int_{\alpha}^{\beta}|a-t|^{n+1} h^{(n+1)}(t) \mathrm{d} t & \leq \sup \left\{h^{(n+1)}(t), t \in[\alpha, \beta]\right\} \int_{\alpha}^{\beta}|a-t|^{n+1} \mathrm{~d} t \\
& =\frac{(\beta-\alpha)^{n+2}}{n+2}\left\|h^{(n+1)}\right\|_{+\infty}=\frac{\left|a-h^{-1}(b)\right|^{n+2}}{n+2}\left\|h^{(n+1)}\right\|_{+\infty},
\end{aligned}
$$

and, by Hölder's integral inequality (2.3),

$$
\begin{aligned}
& \int_{\alpha}^{\beta}|a-t|^{n+1} h^{(n+1)}(t) \mathrm{d} t \\
& \quad \leq\left(\int_{\alpha}^{\beta}|a-t|^{p(n+1)} \mathrm{d} t\right)^{1 / p}\left(\int_{\alpha}^{\beta}\left[h^{(n+1)}(t)\right]^{q} \mathrm{~d} t\right)^{1 / q} \\
& \quad=\left[\frac{(\beta-\alpha)^{p(n+1)+1}}{p(n+1)+1}\right]^{1 / p}\left\|h^{(n+1)}\right\|_{q}=\left[\frac{\left|a-h^{-1}(b)\right|^{p(n+1)+1}}{p(n+1)+1}\right]^{1 / p}\left\|h^{(n+1)}\right\|_{q}
\end{aligned}
$$

for $p>1$ and $\frac{1}{p}+\frac{1}{q}=1$, it follows that

1. when $b>h(a)$ and $n=2 \ell$ for $\ell \geq 0$ or when $b<h(a)$, we have

$$
\begin{aligned}
& \int_{0}^{a} h(x) \mathrm{d} x+\int_{0}^{b} h^{-1}(x) \mathrm{d} x-a b \\
& \quad \leq \sum_{k=1}^{n} h^{(k)}\left(h^{-1}(b)\right) \frac{\left[a-h^{-1}(b)\right]^{k+1}}{(k+1) !}+\frac{C_{p, n}}{(n+1) !}\left\|h^{(n+1)}\right\|_{q}
\end{aligned}
$$

2. when $b>h(a)$ and $n=2 \ell+1$ for $\ell \geq 0$, we have

$$
\begin{aligned}
& \int_{0}^{a} h(x) \mathrm{d} x+\int_{0}^{b} h^{-1}(x) \mathrm{d} x-a b \\
& \quad \geq \sum_{k=1}^{n} h^{(k)}\left(h^{-1}(b)\right) \frac{\left[a-h^{-1}(b)\right]^{k+1}}{(k+1) !}-\frac{C_{p, n}}{(n+1) !}\left\|h^{(n+1)}\right\|_{q}
\end{aligned}
$$

where $\frac{1}{p}+\frac{1}{q}=1$ for $1<p, q<\infty$, or $(p, q)=(+\infty, 1)$, or $(p, q)=(1,+\infty)$.

On the other hand, since

$$
\int_{\alpha}^{\beta}|a-t|^{n+1} h^{(n+1)}(t) \mathrm{d} t \geq 0 \int_{\alpha}^{\beta} h^{(n+1)}(t) \mathrm{d} t=0\left\|h^{(n+1)}\right\|_{1},
$$




$$
\begin{aligned}
\int_{\alpha}^{\beta}|a-t|^{n+1} h^{(n+1)}(t) \mathrm{d} t & \geq \inf \left\{h^{(n+1)}(t), t \in[\alpha, \beta]\right\} \int_{\alpha}^{\beta}|a-t|^{n+1} \mathrm{~d} t \\
& =\frac{(\beta-\alpha)^{n+2}}{n+2}\left\|h^{(n+1)}\right\|_{-\infty}=\frac{\left|a-h^{-1}(b)\right|^{n+2}}{n+2}\left\|h^{(n+1)}\right\|_{-\infty},
\end{aligned}
$$

and, by the reversed version of Hölder's integral inequality (2.3),

$$
\begin{aligned}
& \int_{\alpha}^{\beta}|a-t|^{n+1} h^{(n+1)}(t) \mathrm{d} t \\
& \quad \geq\left(\int_{\alpha}^{\beta}|a-t|^{p(n+1)} \mathrm{d} t\right)^{1 / p}\left(\int_{\alpha}^{\beta}\left[h^{(n+1)}(t)\right]^{q} \mathrm{~d} t\right)^{1 / q} \\
& \quad=\left[\frac{(\beta-\alpha)^{p(n+1)+1}}{p(n+1)+1}\right]^{1 / p}\left\|h^{(n+1)}\right\|_{q}=\left[\frac{\left|a-h^{-1}(b)\right|^{p(n+1)+1}}{p(n+1)+1}\right]^{1 / p}\left\|h^{(n+1)}\right\|_{q}
\end{aligned}
$$

for $p<1, p \neq 0$, and $\frac{1}{p}+\frac{1}{q}=1$, it follows that

1. when $b>h(a)$ and $n=2 \ell$ for $\ell \geq 0$ or when $b<h(a)$, we have

$$
\begin{aligned}
& \int_{0}^{a} h(x) \mathrm{d} x+\int_{0}^{b} h^{-1}(x) \mathrm{d} x-a b \\
& \quad \geq \sum_{k=1}^{n} h^{(k)}\left(h^{-1}(b)\right) \frac{\left[a-h^{-1}(b)\right]^{k+1}}{(k+1) !}+\frac{C_{p, n}}{(n+1) !}\left\|h^{(n+1)}\right\|_{q} ;
\end{aligned}
$$

2. when $b>h(a)$ and $n=2 \ell+1$ for $\ell \geq 0$, we have

$$
\begin{aligned}
& \int_{0}^{a} h(x) \mathrm{d} x+\int_{0}^{b} h^{-1}(x) \mathrm{d} x-a b \\
& \quad \leq \sum_{k=1}^{n} h^{(k)}\left(h^{-1}(b)\right) \frac{\left[a-h^{-1}(b)\right]^{k+1}}{(k+1) !}-\frac{C_{p, n}}{(n+1) !}\left\|h^{(n+1)}\right\|_{q} ;
\end{aligned}
$$

where $p<1$ and $p \neq 0$ with $\frac{1}{p}+\frac{1}{q}=1$, or $(p, q)=(-\infty, 1)$, or $(p, q)=(1,-\infty)$. The proof of Theorem 3.2 is complete.

Remark 3.2 Taking $n=0$ in Theorem 3.2 leads to [6, Theorem 2.1] mentioned above.

Theorem 3.3 Let $n \geq 0$ and $h(x) \in C^{n+1}[0, c]$ such that $h(0)=0$ and $h(x)$ is strictly increasing on $[0, c]$ for $c>0$, let $h^{-1}$ be the inverse function of $h$, let $a \in[0, c]$ and $b \in[0, h(c)]$, and let $\ell \geq 0$ be an integer. Then

1. when

(a) either $h(a)>b$ and $h^{(n+1)}(x)$ is increasing on $[\alpha, \beta]$;

(b) or $h(a)<b, h^{(n+1)}(x)$ is increasing on $[\alpha, \beta]$, and $n=2 \ell+1$;

(c) or $h(a)<b, h^{(n+1)}(x)$ is decreasing on $[\alpha, \beta]$, and $n=2 \ell$;

the inequality

$$
\begin{aligned}
& \int_{0}^{a} h(x) \mathrm{d} x+\int_{0}^{b} h^{-1}(x) \mathrm{d} x-a b-\sum_{k=1}^{n} h^{(k)}\left(h^{-1}(b)\right) \frac{\left[a-h^{-1}(b)\right]^{k+1}}{(k+1) !} \\
& \quad \leq \frac{\left[a-h^{-1}(b)\right]^{n+1}}{(n+2) !}\left[h^{(n)}(a)-h^{(n)}\left(h^{-1}(b)\right)\right]
\end{aligned}
$$

is valid; 
2. when
(a) either $h(a)>b$ and $h^{(n+1)}(x)$ is decreasing on $[\alpha, \beta]$;
(b) or $h(a)<b, h^{(n+1)}(x)$ is increasing on $[\alpha, \beta]$, and $n=2 \ell$;
(c) or $h(a)<b, h^{(n+1)}(x)$ is decreasing on $[\alpha, \beta]$, and $n=2 \ell+1$;
inequality (3.5) is reversed;

where $\alpha, \beta$ are defined as in (1.3).

Proof When $a>h^{-1}(b)$ and $h^{(n+1)}(x)$ is increasing, applying Lemma 2.4 gives

$$
\begin{aligned}
& \int_{h^{-1}(b)}^{a}(a-t)^{n+1} h^{(n+1)}(t) \mathrm{d} t \\
& \quad \leq \frac{1}{a-f^{-1}(b)} \int_{h^{-1}(b)}^{a}(a-t)^{n+1} \mathrm{~d} t \int_{h^{-1}(b)}^{a} h^{(n+1)}(t) \mathrm{d} t \\
& \quad=\frac{\left[a-h^{-1}(b)\right]^{n+1}}{n+2}\left[h^{(n)}(a)-h^{(n)}\left(h^{-1}(b)\right)\right]
\end{aligned}
$$

when $a>h^{-1}(b)$ and $h^{(n+1)}(x)$ is decreasing, the above inequality is reversed.

When $a<h^{-1}(b), h^{(n+1)}(x)$ is increasing, and $n=2 \ell$ for $\ell \geq 0$, applying Lemma 2.4 reveals

$$
\begin{aligned}
& \int_{h^{-1}(b)}^{a}(a-t)^{n+1} h^{(n+1)}(t) \mathrm{d} t \\
& \quad=(-1)^{n} \int_{a}^{h^{-1}(b)}(t-a)^{n+1} h^{(n+1)}(t) \mathrm{d} t \\
& \quad \geq(-1)^{n} \frac{\left[h^{-1}(b)-a\right]^{n+1}}{n+2}\left[h^{(n)}\left(h^{-1}(b)\right)-h^{(n)}(a)\right] \\
& =\frac{\left[a-h^{-1}(b)\right]^{n+1}}{n+2}\left[h^{(n)}(a)-h^{(n)}\left(h^{-1}(b)\right)\right] ;
\end{aligned}
$$

when $a<h^{-1}(b), h^{(n+1)}(x)$ is increasing, and $n=2 \ell+1$ for $\ell \geq 0$, inequality (3.6) is reversed.

By similar argument, we obtain that

1. when $a<h^{-1}(b), h^{(n+1)}(x)$ is decreasing, and $n=2 \ell$ for $\ell \geq 0$, inequality (3.6) is reversed;

2. when $a<h^{-1}(b), h^{(n+1)}(x)$ is decreasing, and $n=2 \ell+1$ for $\ell \geq 0$, inequality (3.6) is valid.

Substituting these inequalities into (3.4) and simplifying lead to inequality (3.5) and its reversed version for all cases. The proof of Theorem 3.3 is complete.

Remark 3.3 Taking $n=0$ in (3.5) derives the right inequality in (1.4).

Theorem 3.4 Let $h(x) \in C^{n+1}[0, c]$ such that $h(0)=0$ and $h(x)$ is strictly increasing on $[0, c]$ for $c>0$, let $h^{-1}$ be the inverse function of $h$, and let $a \in[0, c]$ and $b \in[0, h(c)]$. If $h^{(n+1)}(x)$ is convex on $[\alpha, \beta]$, where $\alpha, \beta$ are defined as in (1.3), then 
1. when $h(a)>b$ or when $h(a)<b$ and $n=2 \ell$, we have

$$
\begin{aligned}
& \frac{\left[a-h^{-1}(b)\right]^{n+2}}{n+2} h^{(n+1)}\left(\frac{a+(n+2) h^{-1}(b)}{n+3}\right) \\
& \leq \int_{0}^{a} h(x) \mathrm{d} x+\int_{0}^{b} h^{-1}(x) \mathrm{d} x-a b-\sum_{k=1}^{n} h^{(k)}\left(h^{-1}(b)\right) \frac{\left[a-h^{-1}(b)\right]^{k+1}}{(k+1) !} \\
& \quad \leq\left[a-h^{-1}(b)\right]^{n+2} \frac{h^{(n+1)}(a)+(n+2) h^{(n+1)}\left(h^{-1}(b)\right)}{(n+3) !}
\end{aligned}
$$

2. when $h(a)<b$ and $n=2 \ell+1$, the double inequality (3.7) is reversed;

where $\ell \geq 0$ is an integer. If $h^{(n+1)}(x)$ is concave on $[\alpha, \beta]$, all the above inequalities are reversed for all corresponding cases.

Proof By substitution of variables, we have

$$
\begin{aligned}
& \int_{h^{-1}(b)}^{a}(a-t)^{n+1} h^{(n+1)}(t) \mathrm{d} t \\
& \quad=\left[a-h^{-1}(b)\right]^{n+2} \int_{0}^{1}(1-s)^{n+1} h^{(n+1)}\left(s a+(1-s) h^{-1}(b)\right) \mathrm{d} s .
\end{aligned}
$$

Applying inequality (2.5) to the convex function $h^{(n+1)}(x)$ yields that

1. when $a>h^{-1}(b)$, we have

$$
\begin{aligned}
& \int_{h^{-1}(b)}^{a}(a-t)^{n+1} h^{(n+1)}(t) \mathrm{d} t \\
& \quad \leq\left[a-h^{-1}(b)\right]^{n+2} \int_{0}^{1}(1-s)^{n+1}\left[s h^{(n+1)}(a)+(1-s) h^{(n+1)}\left(h^{-1}(b)\right)\right] \mathrm{d} s \\
& \quad=\left[a-h^{-1}(b)\right]^{n+2} \frac{h^{(n+1)}(a)+(n+2) h^{(n+1)}\left(h^{-1}(b)\right)}{(n+2)(n+3)}
\end{aligned}
$$

2. when $a<h^{-1}(b)$ and $n=2 \ell$, the above inequality is still valid;

3. when $a<h^{-1}(b)$ and $n=2 \ell+1$, the above inequality is reversed.

Substituting these inequalities into (3.4) and simplifying lead to the right inequality in (3.7) and its reversed version for all cases.

Applying inequality (2.6) to the convex function $h^{(n+1)}(x)$ shows that

1. when $a>h^{-1}(b)$, we have

$$
\begin{aligned}
& \int_{h^{-1}(b)}^{a}(a-t)^{n+1} h^{(n+1)}(t) \mathrm{d} t \\
& \quad \geq\left[\int_{h^{-1}(b)}^{a}(a-t)^{n+1} \mathrm{~d} t\right] h^{(n+1)}\left(\frac{\int_{h^{-1}(b)}^{a}(a-t)^{n+1} t \mathrm{~d} t}{\int_{h^{-1}(b)}^{a}(a-t)^{n+1} \mathrm{~d} t}\right) \\
& \quad=\frac{\left[a-h^{-1}(b)\right]^{n+2}}{n+2} h^{(n+1)}\left(\frac{a+(n+2) h^{-1}(b)}{n+3}\right) ;
\end{aligned}
$$

2. when $a<h^{-1}(b)$ and $n=2 \ell$, the above inequality is still valid;

3. when $a<h^{-1}(b)$ and $n=2 \ell$, the above inequality is reversed. 
Substituting these related inequalities into (3.4) and rearranging result in the left inequality in (3.7) and its reversed version for all cases.

For the concave function $h^{(n+1)}(x)$, one can derive everything similarly. The proof of Theorem 3.4 is complete.

Theorem 3.5 Let $n \geq 0$ and $h(x) \in C^{n+1}[0, c]$ such that $h(0)=0$ and $h(x)$ is strictly increasing on $[0, c]$ for $c>0$, let $h^{-1}$ be the inverse function of $h$, let $a \in[0, c]$ and $b \in[0, h(c)]$, and let $h^{(n+1)}(x)$ be nonnegative and convex on $[\alpha, \beta]$, where $\alpha, \beta$ are defined as in (1.3). If $h(a)>b$, then

$$
\begin{aligned}
& \frac{\left[a-h^{-1}(b)\right]^{n+2}}{(n+1) !}\left[\frac{1}{2^{n}} h^{(n+1)}\left(\frac{a+h^{-1}(b)}{2}\right)-\frac{2 h^{(n+1)}(a)+h^{(n+1)}\left(h^{-1}(b)\right)}{6}\right] \\
& \leq \int_{0}^{a} h(x) \mathrm{d} x+\int_{0}^{b} h^{-1}(x) \mathrm{d} x-a b-\sum_{k=1}^{n} h^{(k)}\left(h^{-1}(b)\right) \frac{\left[a-h^{-1}(b)\right]^{k+1}}{(k+1) !} \\
& \leq \frac{\left[a-h^{-1}(b)\right]^{n+2}}{(n+1) !} \frac{h^{(n+1)}(a)+2 h^{(n+1)}\left(h^{-1}(b)\right)}{6} .
\end{aligned}
$$

If $h(a)<b$ and $n=2 \ell$ for $\ell \geq 0$, then

$$
\begin{aligned}
& \frac{\left[h^{-1}(b)-a\right]^{n+2}}{(n+1) !}\left[\frac{1}{2^{n}} h^{(n+1)}\left(\frac{a+h^{-1}(b)}{2}\right)-\frac{2 h^{(n+1)}(a)+h^{(n+1)}\left(h^{-1}(b)\right)}{6}\right] \\
& \leq \int_{0}^{a} h(x) \mathrm{d} x+\int_{0}^{b} h^{-1}(x) \mathrm{d} x-a b-\sum_{k=1}^{n} h^{(k)}\left(h^{-1}(b)\right) \frac{\left[a-h^{-1}(b)\right]^{k+1}}{(k+1) !} \\
& \leq \frac{\left[h^{-1}(b)-a\right]^{n+2}}{(n+1) !} \frac{h^{(n+1)}(a)+2 h^{(n+1)}\left(h^{-1}(b)\right)}{6} .
\end{aligned}
$$

If $a<h^{-1}(b)$ and $n=2 \ell+1$ for $\ell \geq 0$, the double inequality (3.9) is reversed.

Proof When $a>h^{-1}(b)$, employing the double inequality (2.7) gives

$$
\begin{aligned}
2[ & \left.\frac{a-h^{-1}(b)}{2}\right]^{n+1} h^{(n+1)}\left(\frac{a+h^{-1}(b)}{2}\right) \\
& -\frac{\left[a-h^{-1}(b)\right]^{n+1} h^{(n+1)}\left(h^{-1}(b)\right)}{6}-\frac{\left[a-h^{-1}(b)\right]^{n+1} h^{(n+1)}(a)}{3} \\
& \leq \frac{1}{a-h^{-1}(b)} \int_{h^{-1}(b)}^{a}(a-t)^{n+1} h^{(n+1)}(t) \mathrm{d} t \\
& \leq \frac{\left[a-h^{-1}(b)\right]^{n+1} h^{(n+1)}\left(h^{-1}(b)\right)}{3}+\frac{\left[a-h^{-1}(b)\right]^{n+1} h^{(n+1)}(a)}{6} .
\end{aligned}
$$

When $a<h^{-1}(b)$, it is clear that

$$
\int_{h^{-1}(b)}^{a}(a-t)^{n+1} h^{(n+1)}(t) \mathrm{d} t=(-1)^{n} \int_{a}^{h^{-1}(b)}(t-a)^{n+1} h^{(n+1)}(t) \mathrm{d} t
$$


If $a<h^{-1}(b)$ and $n=2 \ell$, utilizing the double inequality (2.7) gives

$$
\begin{aligned}
2[ & \left.\frac{h^{-1}(b)-a}{2}\right]^{n+1} h^{(n+1)}\left(\frac{a+h^{-1}(b)}{2}\right) \\
& -\frac{\left[h^{-1}(b)-a\right]^{n+1} h^{(n+1)}\left(h^{-1}(b)\right)}{6}-\frac{\left[h^{-1}(b)-a\right]^{n+1} h^{(n+1)}(a)}{3} \\
& \leq \frac{1}{h^{-1}(b)-a} \int_{a}^{h^{-1}(b)}(t-a)^{n+1} h^{(n+1)}(t) \mathrm{d} t \\
& \leq \frac{\left[h^{-1}(b)-a\right]^{n+1} h^{(n+1)}\left(h^{-1}(b)\right)}{3}+\frac{\left[h^{-1}(b)-a\right]^{n+1} h^{(n+1)}(a)}{6} .
\end{aligned}
$$

If $a<h^{-1}(b)$ and $n=2 \ell+1$, the above double inequality is reversed.

Substituting these related inequalities into (3.4) and rearranging conclude the double inequalities (3.8) and (3.9). The proof of Theorem 3.5 is complete.

\section{Applications and examples}

As applications of Theorems 3.1 to 3.5, we now estimate several concrete definite integrals, including a definite integral of $e^{-1 / x}$, the exponential integral $\operatorname{Ei}(x)$, and the logarithmic integral li $(x)$.

Example 4.1 By taking $h(x)=\sqrt[4]{x^{4}+1}-1, a=3$, and $b=2$ in Theorem 1.2, it was obtained in [4] that

$$
\begin{aligned}
9+\frac{4 \sqrt[4]{125}}{27}[3-2 \sqrt[4]{5}]^{2} & =9.000042866 \ldots \\
& <\int_{0}^{3} \sqrt[4]{x^{4}+1} \mathrm{~d} x+\int_{1}^{3} \sqrt[4]{x^{4}-1} \mathrm{~d} x \\
& <9+\frac{27}{2 \sqrt[4]{82^{3}}}(3-2 \sqrt[4]{5})^{2}=9.000042871 \ldots
\end{aligned}
$$

The difference between the upper and lower bounds is $0.00000000490079353 \ldots$.

In $[5,6]$, by Theorems 1.3 and 1.4 , the above double inequality was improved as

$$
9.00004286805 \ldots<\int_{0}^{3} \sqrt[4]{x^{4}+1} \mathrm{~d} x+\int_{1}^{3} \sqrt[4]{x^{4}-1} \mathrm{~d} x<9.000042868057 \ldots
$$

It is easy to see that $h^{-1}(x)=\left[(x+1)^{4}-1\right]^{1 / 4}$. Straightforward computation gives

$$
h^{\prime}(x)=\frac{x^{3}}{\left(x^{4}+1\right)^{3 / 4}}, \quad h^{\prime \prime}(x)=\frac{3 x^{2}}{\left(x^{4}+1\right)^{7 / 4}}, \quad h^{\prime \prime \prime}(x)=\frac{x\left(6-15 x^{4}\right)}{\left(x^{4}+1\right)^{11 / 4}}
$$

Since $h^{-1}(2)=80^{1 / 4}=2.990 \ldots<3$, then $(\alpha, \beta)=\left(80^{1 / 4}, 3\right)$. The third derivative $h^{\prime \prime \prime}(x)$ has two real nonzero zeros $\pm\left(\frac{2}{5}\right)^{1 / 4}= \pm 0.79527 \ldots$. This implies that the third derivative $h^{\prime \prime \prime}(x)$ is negative and $h^{\prime \prime}(x)$ is concave on the closed interval $[\alpha, \beta]$. Substituting these data into 
the reversed version of inequality (3.7) yields

$$
\begin{aligned}
& \frac{\left(3-80^{1 / 4}\right)^{3}}{3} h^{\prime \prime}\left(\frac{3+380^{1 / 4}}{4}\right) \\
& \geq \int_{0}^{3} \sqrt[4]{x^{4}+1} \mathrm{~d} x-3+\int_{1}^{3} \sqrt[4]{x^{4}-1} \mathrm{~d} x-6-h^{\prime}\left(80^{1 / 4}\right) \frac{\left(3-80^{1 / 4}\right)^{2}}{2 !} \\
& \geq\left(3-80^{1 / 4}\right)^{3} \frac{h^{\prime \prime}(3)+3 h^{\prime \prime}\left(80^{1 / 4}\right)}{4 !}
\end{aligned}
$$

which can be rewritten as

$$
\begin{aligned}
& \frac{\left(3-80^{1 / 4}\right)^{3}}{3} \frac{3072(\sqrt[4]{95} \sqrt{2}+3)^{2}}{\left[(\sqrt[4]{95} \sqrt{2}+3)^{4}+256\right]^{7 / 4}}+9+\frac{8 \times 5^{3 / 4}}{27} \frac{\left(3-80^{1 / 4}\right)^{2}}{2 !} \\
& =9.0000428983186013 \ldots \geq \int_{0}^{3} \sqrt[4]{x^{4}+1} \mathrm{~d} x+\int_{1}^{3} \sqrt[4]{x^{4}-1} \mathrm{~d} x \\
& \geq \frac{\left(3-80^{1 / 4}\right)^{3}}{4 !}\left(\frac{27}{82^{7 / 4}}+3 \times \frac{4 \sqrt{5}}{729}\right)+9+\frac{8 \times 5^{3 / 4}}{27} \frac{\left(3-80^{1 / 4}\right)^{2}}{2 !} \\
& =9.0000428680640760 \ldots
\end{aligned}
$$

This lower estimation is better than all before.

Example 4.2 Let $h(x)=e^{-1 / x}$ for $x>0$ and $h(0)=0$. Then $h^{-1}(x)=-\frac{1}{\ln x}$ for $x \in(0,1)$ and $h^{-1}(0)=0$. The function $h(x)=e^{-1 / x}$ plays an indispensable role in the proof of the existence of partitions of unity in differential geometry [30] and is a generating function of the Lah numbers in combinatorics [13-18, 20, 22].

Direct computation gives

$$
h^{\prime}(x)=\frac{e^{-1 / x}}{x^{2}} \quad \text { and } \quad h^{\prime \prime}(x)=\frac{e^{-1 / x}(1-2 x)}{x^{4}} \text {. }
$$

This means that the function $h^{\prime}(x)$ is increasing on $\left(0, \frac{1}{2}\right]$ and decreasing on $\left[\frac{1}{2}, \infty\right)$.

Choosing $a=b=\frac{1}{2}$ means that $a<h^{-1}\left(\frac{1}{2}\right)=\frac{1}{\ln 2}=1.44 \ldots$ and $(\alpha, \beta)=\left(\frac{1}{2}, \frac{1}{\ln 2}\right)$. Then it follows from the double inequality (1.4) that

$$
\begin{aligned}
& \frac{1}{4}+\left(\frac{1}{2}-\frac{1}{\ln 2}\right)\left[\frac{1}{e^{\frac{1}{2}\left(\frac{1}{2}+\frac{1}{\ln 2}\right)}}-\frac{1}{2}\right] \\
& =0.364469045537996606 \ldots \\
& \leq \int_{0}^{1 / 2} \frac{1}{e^{1 / x}} \mathrm{~d} x-\int_{0}^{1 / 2} \frac{1}{\ln x} \mathrm{~d} x=\operatorname{Ei}(-2)-\operatorname{li}\left(\frac{1}{2}\right)+\frac{1}{2 e^{2}} \\
& \leq \frac{1}{4}+\frac{1}{2}\left(\frac{1}{2}-\frac{1}{\ln 2}\right)\left(\frac{1}{e^{2}}-\frac{1}{2}\right)=0.421883810040011829 \ldots \text {, }
\end{aligned}
$$

where

$$
\operatorname{Ei}(x)=-\int_{-x}^{\infty} \frac{e^{-t}}{t} \mathrm{~d} t \quad \text { and } \quad \operatorname{li}(x)=\int_{0}^{x} \frac{\mathrm{d} t}{\ln t}
$$

are respectively called the exponential integral and the logarithmic integral. 
Example 4.3 Let $h(x)=e^{x^{2}}-1$ for $x \geq 0$. Then $h^{-1}(x)=\sqrt{\ln (1+x)}$ for $x \geq 0$.

In [21, Sect. 1.4], it was computed that

$$
h^{(n)}(x)=\left(e^{x^{2}}\right)^{(n)}=e^{x^{2}} \frac{n !}{(2 x)^{n}} \sum_{k=0}^{n}\left(\begin{array}{c}
k \\
n-k
\end{array}\right) \frac{(2 x)^{2 k}}{k !}, \quad n \geq 1 .
$$

This implies that the function $h(x)$ is absolutely monotonic, that is, $h^{(n)}(x) \geq 0$ for all $n \geq 0$, on $(0, \infty)$. For more information on absolutely monotonic functions, please refer to [3] and closely related references therein.

Choosing $a=x$ and $b=x^{2}$ for $x>0$ implies that $h^{-1}\left(x^{2}\right)=\ln ^{1 / 2}\left(1+x^{2}\right)<x$ for all $x>0$. Applying these data to the double inequality (3.8), we arrive at

$$
\begin{aligned}
& \frac{\left[x-\ln ^{1 / 2}\left(1+x^{2}\right)\right]^{n+2}}{(n+1) !}\left[\frac{1}{2^{n}} h^{(n+1)}\left(\frac{x+\ln ^{1 / 2}\left(1+x^{2}\right)}{2}\right)\right. \\
& \left.\quad-\frac{2 h^{(n+1)}(x)+h^{(n+1)}\left(\ln ^{1 / 2}\left(1+x^{2}\right)\right)}{6}\right] \\
& \leq \int_{0}^{x} e^{t^{2}} \mathrm{~d} t+\int_{0}^{x^{2}} \sqrt{\ln (1+t)} \mathrm{d} t-x-x^{3} \\
& \quad-\sum_{k=1}^{n} h^{(k)}\left(\ln ^{1 / 2}\left(1+x^{2}\right)\right) \frac{\left[x-\ln ^{1 / 2}\left(1+x^{2}\right)\right]^{k+1}}{(k+1) !} \\
& \leq \frac{\left[x-\ln ^{1 / 2}\left(1+x^{2}\right)\right]^{n+2}}{(n+1) !} \frac{h^{(n+1)}(x)+2 h^{(n+1)}\left(\ln ^{1 / 2}\left(1+x^{2}\right)\right)}{6}
\end{aligned}
$$

for $n \geq 0$ and $x>0$. In particular, letting $n=1$ derives

$$
\begin{aligned}
& \frac{\left[x-\ln ^{1 / 2}\left(1+x^{2}\right)\right]^{3}}{2}\left[\frac{1}{2} h^{\prime \prime}\left(\frac{x+\ln ^{1 / 2}\left(1+x^{2}\right)}{2}\right)\right. \\
& \left.\quad-\frac{2 h^{\prime \prime}(x)+h^{\prime \prime}\left(\ln ^{1 / 2}\left(1+x^{2}\right)\right)}{6}\right] \\
& \leq \int_{0}^{x} e^{t^{2}} \mathrm{~d} t+\int_{0}^{x^{2}} \sqrt{\ln (1+t)} \mathrm{d} t-x-x^{3} \\
& \quad-h^{\prime}\left(\ln ^{1 / 2}\left(1+x^{2}\right)\right) \frac{\left[x-\ln ^{1 / 2}\left(1+x^{2}\right)\right]^{2}}{2} \\
& \leq \frac{\left[x-\ln ^{1 / 2}\left(1+x^{2}\right)\right]^{3}}{2} \frac{h^{\prime \prime}(x)+2 h^{\prime \prime}\left(\ln ^{1 / 2}\left(1+x^{2}\right)\right)}{6} .
\end{aligned}
$$

Further taking $x=1$ results in

$$
\begin{aligned}
& \frac{\left(1-\ln ^{1 / 2} 2\right)^{3}}{2}\left[\frac{1}{2} h^{\prime \prime}\left(\frac{1+\ln ^{1 / 2} 2}{2}\right)-\frac{2 h^{\prime \prime}(1)+h^{\prime \prime}\left(\ln ^{1 / 2} 2\right)}{6}\right]+h^{\prime}\left(\ln ^{1 / 2} 2\right) \frac{\left(1-\ln ^{1 / 2} 2\right)^{2}}{2}+2 \\
& \leq \int_{0}^{1} e^{t^{2}} \mathrm{~d} t+\int_{0}^{1} \sqrt{\ln (1+t)} \mathrm{d} t \\
& \leq \frac{\left(1-\ln ^{1 / 2} 2\right)^{3}}{2} \frac{h^{\prime \prime}(1)+2 h^{\prime \prime}\left(\ln ^{1 / 2} 2\right)}{6}+h^{\prime}\left(\ln ^{1 / 2} 2\right) \frac{\left(1-\ln ^{1 / 2} 2\right)^{2}}{2}+2,
\end{aligned}
$$


which can be numerically computed as

$$
2.044751320 \ldots \leq \int_{0}^{1} e^{t^{2}} \mathrm{~d} t+\int_{0}^{1} \sqrt{\ln (1+t)} \mathrm{d} t \leq 2.060536019 \ldots
$$

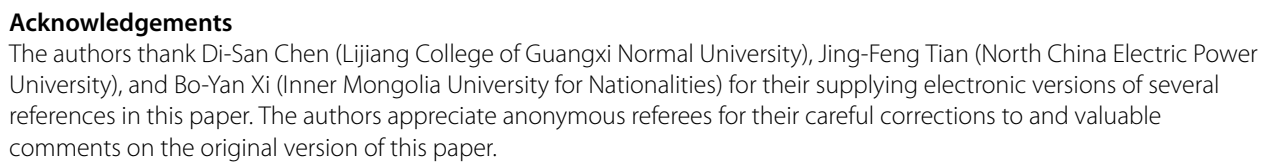

Funding

Not applicable.

Availability of data and materials

Not applicable.

\section{Competing interests}

The authors declare that they have no competing interests.

\section{Authors' contributions}

All authors contributed equally to the manuscript and read and approved the final manuscript.

\section{Author details}

${ }^{1}$ College of Mathematical Sciences, Dezhou University, Dezhou, Shandong, China. ${ }^{2}$ School of Mathematics and Informatics, Henan Polytechnic University, Jiaozuo, China. ${ }^{3}$ School of Mathematical Sciences, Tianjin Polytechnic University, Tianjin, China. ${ }^{4}$ College of Mathematics, Inner Mongolia University for Nationalities, Tongliao, China.

\section{Publisher's Note}

Springer Nature remains neutral with regard to jurisdictional claims in published maps and institutional affiliations.

\section{Received: 14 May 2019 Accepted: 30 August 2019 Published online: 11 September 2019}

\section{References}

1. Apostol, T.M.: Calculus, Vol. I: One-Variable Calculus, with an Introduction to Linear Algebra, 2nd edn. Blaisdell, Waltham (1967)

2. Apostol, T.M.: Mathematical Analysis, 2nd edn. Addison-Wesley, Reading (1974)

3. Guo, B.-N., Qi, F.: A property of logarithmically absolutely monotonic functions and the logarithmically complete monotonicity of a power-exponential function. UPB Sci. Bull., Ser. A, Appl. Math. Phys. 72(2), 21-30 (2010)

4. Hoorfar, A., Qi, F.: A new refinement of Young's inequality. Math. Inequal. Appl. 11(4), 689-692 (2008). https://doi.org/10.7153/mia-11-58

5. Jakšetić, J., Pečarić, J.: An estimation of Young inequality. Asian-Eur. J. Math. 2(4), 593-604 (2009). https://doi.org/10.1142/S1793557109000509

6. Jakšetić, J., Pečarić, J.: A note on Young inequality. Math. Inequal. Appl. 13(1), 43-48 (2010). https://doi.org/10.7153/mia-13-03

7. Mercer, P.R.: Error terms for Steffensen's, Young's and Chebychev's inequalities. J. Math. Inequal. 2(4), 479-486 (2008). https://doi.org/10.7153/jmi-02-43

8. Mercer, P.R.: Techniques of integration. In: More Calculus of a Single Variable, Chap. 11. Undergraduate Texts in Mathematics. Springer, New York (2014). https://doi.org/10.1007/978-1-4939-1926-0_11.

9. Mitrinović, D.S.: Analytic Inequalities, in Cooperation with P. M. Vasić. Die Grundlehren der mathematischen Wissenschaften, vol. 165. Springer, New York (1970)

10. Mitrinović, D.S., Pečarić, J.E., Fink, A.M.: Classical and New Inequalities in Analysis. Kluwer Academic, Dordrecht (1993). https://doi.org/10.1007/978-94-017-1043-5

11. Olver, F.W.J., Lozier, D.W., Boisvert, R.F., Clark, C.W. (eds.): NIST Handbook of Mathematical Functions. Cambridge University Press, New York (2010). http://dlmf.nist.gov/

12. Pachpatte, B.G.: On some inequalities for convex functions. RGMIA Res. Rep. Collect. 6, Suppl., Art. 1 (2003). http://rgmia.org/v6(E).php

13. Qi, F.: Explicit formulas for computing Bernoulli numbers of the second kind and Stirling numbers of the first kind. Filomat 28(2), 319-327 (2014). https://doi.org/10.2298/FIL14023190

14. Qi, F.: Derivatives of tangent function and tangent numbers. Appl. Math. Comput. 268, 844-858 (2015). https://doi.org/10.1016/j.amc.2015.06.123

15. Qi, F.: Properties of modified Bessel functions and completely monotonic degrees of differences between exponential and trigamma functions. Math. Inequal. Appl. 18(2), 493-518 (2015). https://doi.org/10.7153/mia-18-37

16. Qi, F: An explicit formula for the Bell numbers in terms of the Lah and Stirling numbers. Mediterr. J. Math. 13(5), 2795-2800 (2016). https://doi.org/10.1007/s00009-015-0655-7

17. Qi, F: Diagonal recurrence relations for the Stirling numbers of the first kind. Contrib. Discrete Math. 11, 22-30 (2016). https://doi.org/10515/sy5wh2dx6. http://hdl.handle.net/10515/sy5wh2dx6 
18. Qi, F., Berg, C.: Complete monotonicity of a difference between the exponential and trigamma functions and properties related to a modified Bessel function. Mediterr. J. Math. 10(4), 1685-1696 (2013). https://doi.org/10.1007/s00009-013-0272-2

19. Qi, F., Cui, L.-H., Xu, S.-L.: Some inequalities constructed by Tchebysheff's integral inequality. Math. Inequal. Appl. 2(4), 517-528 (1999). https://doi.org/10.7153/mia-02-42

20. Qi, F., Lim, D., Guo, B.-N.: Explicit formulas and identities for the Bell polynomials and a sequence of polynomials applied to differential equations. Rev. R. Acad. Cienc. Exactas Fís. Nat., Ser. A Mat. 113(1), 1-9 (2019). https://doi.org/10.1007/s13398-017-0427-2

21. Qi, F., Niu, D.-W., Lim, D., Yao, Y.-H.: Special values of the Bell polynomials of the second kind for some sequences and functions. HAL archives (2018). https://hal.archives-ouvertes.fr/hal-01766566

22. Qi, F., Zheng, M.-M.: Explicit expressions for a family of the Bell polynomials and applications. Appl. Math. Comput. 258, 597-607 (2015). https://doi.org/10.1016/j.amc.2015.02.027

23. Sándor, J., Szabó, V.E.S.: On an inequality for the sum of infimums of functions. J. Math. Anal. Appl. 204(3), 646-654 (1996). https://doi.org/10.1006/jmaa.1996.0459

24. Tian, J.-F.: Extension of Hu Ke's inequality and its applications. J. Inequal. Appl. 2011, 77 (2011). https://doi.org/10.1186/1029-242X-2011-77

25. Tian, J.-F., Ha, M.-H.: Properties of generalized sharp Hölder's inequalities. J. Math. Inequal. 11(2), 511-525 (2017) https://doi.org/10.7153/jmi-11-42

26. Wu, Y., Qi, F., Niu, D.-W.: Integral inequalities of Hermite-Hadamard type for the product of strongly logarithmically convex and other convex functions. Maejo Int. J. Sci. Technol. 9(3), 394-402 (2015)

27. Yin, H.-P., Qi, F.: Hermite-Hadamard type inequalities for the product of $(\alpha, m)$-convex functions. J. Nonlinear Sci. Appl. 8(3), 231-236 (2015). https://doi.org/10.22436/jnsa.008.03.07

28. Yin, H.-P., Qi, F.: Hermite-Hadamard type inequalities for the product of $(\alpha, m)$-convex functions. Mo. J. Math. Sci. 27(1), 71-79 (2015). http://projecteuclid.org/euclid.mjms/1449161369

29. Young, W.H.: On classes of summable functions and their Fourier series. Proc. R. Soc. Lond. Ser. A 87, 225-229 (1912). https://doi.org/10.1098/rspa.1912.0076

30. Zhang, X.-J., Qi, F., Li, W.-H.: Properties of three functions relating to the exponential function and the existence of partitions of unity. Int. J. Open Probl. Comput. Sci. Math. 5(3), 122-127 (2012). https://doi.org/10.12816/0006128

\section{Submit your manuscript to a SpringerOpen ${ }^{\circ}$ journal and benefit from:}

- Convenient online submission

- Rigorous peer review

- Open access: articles freely available online

- High visibility within the field

- Retaining the copyright to your article

Submit your next manuscript at $>$ springeropen.com 